\title{
ANALYSE ÉCONOMIQUE DES PRATIQUES DE FIDÉLISATION ${ }^{1}$ \\ Wilfried Sand-Zantman
}

Toulouse School of Economics (IDEI et GREMAQ)

\begin{abstract}
Résumé
Le développement des NTIC et l'ouverture à la concurrence de nouveaux secteurs industriels ont conduit à une généralisation des pratiques de fidélisation et de suivi de la clientèle. Cet article présente l'analyse économique de ces pratiques, de leur impact sur la concurrence et le bien-être des consommateurs. Nous expliquons pourquoi, dans un cadre monopolistique, la fidélisation peut se comprendre comme une façon d'inciter à la consommation, au profit de la firme et parfois également des consommateurs. Nous montrons ensuite que, dans un univers plus concurrentiel, cette pratique tend à intensifier le degré de concurrence effective, au bénéfice quasi-exclusif des consommateurs. Nous discutons enfin de l'impact pro ou anti concurrentiel de pratiques reliées, par exemple les contrats de long terme et les offres de rétention.
\end{abstract}

\section{An Economic Analysis of Loyalty Rewards}

\begin{abstract}
The rise of new information technologies and the opening to competition of previously monopolized sectors have induced more dynamic pricing practices, in particular the development of loyalty rewards. We aim at looking at the economic motivations and impacts of this new trend, in particular with regards to effective competition and consumer welfare. First, we explain that, in a monopolistic setting, loyalty rewards can induce more consumption and benefit both firms and consumers. Then, in a duopolistic setting, we show that behavior-based price discrimination increases effective competition and benefits consumers. At last, we discuss the pro- or anticompetitive impacts of related practices, such as long-term contracting and retention offers.
\end{abstract}

Codes JEL : L1 ; D82 ; D43 ; L40

Mots clefs : fidélisation, concurrence imparfaite, discrimination en prix.

${ }^{1}$ L'auteur remercie Bruno Jullien, Marc Lebourges et Claudia Saavedra pour leurs commentaires, ainsi que Orange-FT Groupe pour son soutien. Les remarques constructives d'un rapporteur anonyme ont également contribué à améliorer une première version de ce travail. 


\section{Introduction}

Les programmes de fidélisation, apparus il y a près d'un siècle suite aux offres de S\&H Green Stamp aux Etats-Unis, ont connu un développement important depuis une vingtaine d'années. En effet, alors que la libéralisation de certains secteurs de l'économie a contraint un nombre croissant d'entreprises à adopter des stratégies commerciales offensives, le développement des Nouvelles Technologies de l'Information et de la Communication (NTIC) a permis la mise en place de nouveaux outils de gestion de la clientèle. Dans des marchés dynamiques, avec de nouveaux outils, les défis classiques posés aux entreprises (Comment conserver les clients passés? Comment conquérir les clients d'un concurrent alors que celui-ci tente de les conserver ?) doivent être repensés. Plus globalement, et au-delà de l'étude des stratégies commerciales, il est fondamental de comprendre l'impact de ces stratégies sur l'intensité concurrentielle et finalement sur le bienêtre des consommateurs. Cet article se propose donc, en se basant sur les avancées récentes de la théorie économique, d'analyser les conséquences positives et normatives des pratiques de fidélisation ${ }^{2}$.

Les stratégies de fidélisation peuvent revêtir des formes diverses. Parfois, les consommateurs passés bénéficient directement de tarifs préférentiels en cas de nouvel achat (ou de réabonnement dans le cas d'un service). II arrive également que les achats répétés permettent de collecter des points qui donnent droit à des tarifs préférentiels au-delà d'un certain nombre de points. Les programmes Frequent Flyers développés par les compagnies aériennes en sont un exemple, tout comme les programmes de fidélité proposés par l'ensemble des grandes surfaces - on parle par analogie de programme Frequent Shoppers. Les entreprises peuvent également proposer initialement des contrats de longue durée qui, en échange bien souvent de services ou de prix avantageux, contraignent les consommateurs à rester fidèles à cette entreprise pendant une durée minimale. C'est le cas en particulier dans le secteur des télécommunications où ce type de pratique n'est pas toujours vu d'un bon œil par les autorités de la concurrence, en particulier lorsqu'elles sont le fait d'une entreprise historiquement dominante sur le marché.

\footnotetext{
${ }^{2}$ Voir le livre de L. Meyer-Waarden [2012] pour une approche « marketing » du problème.
} 
Avantage tarifaire, discrimination en fonction des comportements passés, coupons de réduction et programmes de fidélité sont autant de pratiques variées qui dépendent du contexte, et en grande partie de l'information disponible sur les consommateurs. En effet, mettre en place un ciblage de clientèle, comme les programmes de fidélisation de type Frequent Flyer, nécessite un repérage et un suivi des individus en fonction de leur choix de consommation.

Notons également que, parallèlement aux programmes visant à conserver sa clientèle, se développent des programmes visant à acquérir de nouvelles parts de marché, bien souvent aux dépens de ses concurrents. En effet, en plus des offres commerciales de type "pay-to-stay ", récompensant les consommateurs fidèles, certaines entreprises proposent des offres " pay-toswitch ", visant ici à attirer des nouveaux clients. Dans les services Internet, ou d'abonnement à des chaines payantes (Canal+ en est un exemple), les nouveaux abonnés bénéficient d'avantages supplémentaires par rapport aux anciens clients. Les journaux sur Internet (comme Le Monde) proposent également des offres spécifiques et avantageuses aux nouveaux abonnés.

Pour comprendre les stratégies des entreprises comme leur impact sur les consommateurs, il est utile de raisonner en utilisant le concept économique de coûts de changement, ou switching costs. Ces coûts de changement représentent la difficulté pour un consommateur de passer d'un fournisseur à un autre. Ils peuvent donc constituer un frein à la concurrence. Notons que ces coûts ne sont pas liés à la différence entre les produits proposés par les différentes firmes sur le marché mais dépendent généralement des contraintes techniques et du temps passé par les consommateurs pour changer de fournisseurs ou adapter leurs pratiques de consommation (on peut penser par exemple au passage d'un système Windows à un système Apple). Parfois, les firmes peuvent influencer ces coûts soit directement (absence de portabilité des mobiles, normes et standards particuliers dans le cas des produits audio/video), soit indirectement en attribuant des avantages dont l'importance croît dans le temps et qui disparaissent en cas de changement de fournisseur. Même si des liens existent entre les pratiques de fidélisation et le problème des coûts de changement, les problèmes sont en réalité assez différents. Dans une contribution consacrée aux switching costs, Klemperer [1995] montre que la présence de ces coûts conduit les firmes à arbitrer entre exploiter les clients captifs avec des prix élevés et augmenter sa part de marché avec des prix bas. Mais en considérant des prix uniformes pour tous les consommateurs quels que soient 
leurs profils passés de consommation, Klemperer [1995] ne prend pas vraiment en compte ce qui est sans doute le cœur des phénomènes de fidélisation.

Les analyses empiriques relatives à la profitabilité des pratiques de fidélisation restent peu nombreuses et assez spécifiques à un secteur donné. Idéalement, on voudrait savoir comment ces pratiques affectent le marché et si ces pratiques sont bénéfiques pour les firmes et les consommateurs.

Lederman [2007] est un des rares exemples d'étude détaillée des programmes de fidélisation. ${ }^{3}$ ॥I s'intéresse spécifiquement aux programmes Frequent Flyer proposés par les compagnies aériennes, en essayant de mettre au jour l'impact de tels programmes sur la demande. A partir de données portant sur le marché Nord-Américain, il montre que ces programmes sont effectivement générateurs de demande. Mais ces résultats posent un problème d'interprétation car pour le marché business - le plus sensible à ces programmes -, ce sont bien souvent les entreprises qui payent les billets choisis par leurs employés, ces derniers étant donc particulièrement réactifs aux programmes de Frequent Flyer (dont ils bénéficient) et peu au prix (payé par leur employeur). Notons également que l'étude de Lederman, si elle conclut à une augmentation des prix suite à l'introduction de ces programmes, ne dit rien des profits des firmes mettant en place de tels programmes. ${ }^{4}$

Cet article propose, à partir des contributions de la littérature économique, une réflexion sur différentes facettes des pratiques de fidélisation ou d'acquisition de la base clientèle. Même si les programmes mis en place par les entreprises s'analysent généralement dans un cadre de concurrence, il est fructueux de commencer à les analyser dans un cadre monopolistique, ce qui est

\footnotetext{
${ }^{3}$ De façon connexe, W. Hartmann et B. Viard [2008] montrent, dans le cas des clubs de golf, que les programmes de fidélisation peuvent créer des switching costs mais la question de la profitabilité est mise de côté.

${ }^{4}$ Dans le cas particulier des programmes de Frequent Flyers avec des clients bénéficiant du programme mais ne payant pas directement pour le service, Basso et al. [2009] montrent que si une firme isolée peut avoir intérêt à mettre en place un tel programme, son profit peut en pâtir lorsque toutes les firmes du secteur font de même.
} 
l'objet de la deuxième section. Cela permet de mettre en lumière le rôle des programmes de fidélisation comme outil de tarification et de discrimination des consommateurs. La section 3 discute l'impact de ces programmes dans un cadre concurrentiel. Plus précisément, lorsque les entreprises peuvent différencier leurs offres - et en particulier les prix - en fonction du comportement passé des consommateurs, elles peuvent parfois favoriser les consommateurs passés (la stratégie pay-to-stay) ou bien au contraire les nouveaux consommateurs (stratégie payto-switch). La quatrième section étoffe ces premières analyses en s'intéressant à certaines pratiques courantes en matière de fidélisation. Ainsi, nous verrons que les contrats de long terme, en plus d'être un instrument de discrimination des consommateurs, peuvent avoir un rôle stratégique en permettant à un opérateur dominant de conserver sa position. Nous montrerons également que si certaines formes de fidélisation peuvent conduire à une collusion tacite entre firmes concurrentes, d'autres pratiques de plus en plus courantes (comme les offres de rétention) permettent au contraire d'augmenter la concurrence et le bien-être social. La cinquième section conclura cette contribution.

\section{La fidélisation comme outil de discrimination}

Il s'agit ici d'analyser les programmes de fidélisation comme un outil de tarification visant, pour une firme en monopole, à extraire le maximum de surplus de sa relation avec les consommateurs. Pour que cette analyse fasse sens, il faut bien sûr se mettre dans un cadre dynamique où les consommateurs peuvent, après une première période d'achat, consommer à nouveau le bien. En ce sens, cette analyse s'applique bien aux services ou aux biens de consommation courante, et assez peu aux biens durables. Pourtant, nous verrons que certains problèmes dynamiques des biens durables - en particulier la concurrence dite " coasienne » qu'une firme se fait à elle-même intertemporellement - sont également à l'œuvre dans l'analyse des programmes de fidélisation.

\subsection{Encouragement à l’achat précoce}

Une première approche intéressante consiste à comprendre, dans le cas des achats répétés, pourquoi les clients ayant déjà consommé le bien sont parfois mieux traités, en termes de prix, que ceux qui ne l'ont jamais consommé. Au niveau pratique, ce traitement privilégié peut se traduire 
par la présence d'un prix préférentiel pour le deuxième achat ou encore, de façon équivalente ici, par l'attribution lors du premier achat de coupons de réduction valables lors du deuxième achat. Cette pratique peut paraitre paradoxale car les agents qui achètent le bien de façon répétée sont ceux qui en retirent le plus grand bénéfice et donc pour lesquels il ne semble pas nécessaire de faire un effort commercial.

Pour élucider ce paradoxe, intéressons-nous à une situation où les consommateurs sont susceptibles de consommer un bien de façon répétée ${ }^{5}$. Ce bien a une valeur différente pour chaque consommateur et, avant de l'avoir consommé une première fois, aucun consommateur ne peut savoir si le bien lui convient - et donc s'il serait susceptible de le consommer à nouveau - ou pas. Comme on le voit, le bien (ou le service) peut s'analyser comme un bien d'expérience pour lequel les préférences des consommateurs sont inconnues ex-ante mais stables dans le temps.

Supposons que le monopole puisse distinguer les nouveaux consommateurs des anciens et qu'il ait le droit de proposer des prix différents. Initialement, pour le monopole, tous les consommateurs sont les mêmes à la fois parce que leur historique de consommation est vierge et parce qu'ils ne connaissent pas eux-mêmes leur préférence et donc ont tous la même évaluation de leur propre goût. C'est pourquoi, à la première période, le monopole va choisir un prix tel que tous les consommateurs essayent le bien. Quant au prix de deuxième période, il est choisi en même temps que le prix de première période et ce choix, communiqué ex-ante aux consommateurs potentiels, engage le monopole. Ainsi, un consommateur s'interrogeant sur sa décision d'achat en première période va considérer non seulement le prix de première période, mais également le prix de deuxième période s'il a apprécié le bien. La question pour le monopole est donc celle du choix optimal de ces deux prix, c'est-à-dire de la pondération entre les deux parties du tarif.

On peut montrer que le monopole va proposer un prix de première période plus élevé que celui de deuxième période, ce dernier étant égal au coût marginal de production. En proposant un prix de seconde période égal au coût de production, le monopole maximise le surplus de la relation avec les consommateurs de deuxième période. Comme le surplus laissé en seconde période est élevé, le monopole peut choisir un prix élevé en première période pour extraire ce surplus, et également une partie du surplus lié à la consommation de première période.

\footnotetext{
${ }^{5}$ C'est l'approche utilisée par Crémer [1984].
} 


\section{Les ventes répétées avec biens d'expérience : Illustration numérique}

Pour illustrer plus précisément les idées précédentes, on suppose que le coût marginal de production est égal à $1 / 4$ et que la valeur du bien pour les consommateurs est distribuée de façon uniforme entre 0 et 1 .

Comme les consommateurs n'apprennent leur disposition à payer qu'en consommant, soit ils ne consomment jamais (et obtiennent alors une utilité nulle), soit ils consomment à la première période puis éventuellement à la deuxième si le prix est inférieur à leur évaluation du bien. Si tous les consommateurs achètent le bien à la première période et seuls ceux ayant une utilité supérieure au prix renouvellent leur achat à la deuxième période, le profit du monopole est donné par $\left(p_{1^{-}}\right.$ $1 / 4)+\left(p_{2}-1 / 4\right)\left(1-p_{2}\right)$

Supposons tout d'abord que le même prix est proposé à chaque période, i.e., $p_{1}=p_{2}=p$. Le prix maximisant le profit du monopole est égal à 1 mais ce prix unique doit permettre aux consommateurs d'obtenir un surplus au moins égal à 0 . L'utilité moyenne des consommateurs en première période est donnée par $1 / 2-p$ alors que l'utilité espérée conditionnellement à consommer en deuxième période est $\frac{1}{2}(1-p)^{2}$. Les consommateurs acceptent donc de consommer en première période pour un prix $p$ inférieur ou égal à $2-\sqrt{2}$. Pour ce prix limite, le profit de la firme est égal à $\sqrt{2}\left(\frac{7}{4}-\sqrt{2}\right)$.

Supposons maintenant que les prix en première et deuxième période sont différents. Si la firme choisit de maximiser la valeur créée en deuxième période, elle va proposer le prix efficace, c'est-àdire fixer un prix égal au coût marginal, soit $p_{2}=1 / 4$. Avec ce prix, le nombre de consommateurs bénéficiant du bien augmente ( $\operatorname{car} 1 / 4<2-\sqrt{2}$ ), et le surplus espéré en deuxième période est maintenant donné par $9 / 32$. Pour choisir son prix de première période, la firme va vouloir récupérer ce surplus de deuxième période mais également le surplus lié à la première période. Le monopole va donc choisir le niveau de prix le plus élevé incitant le consommateur à consommer dès la première période, $c^{\prime}$ est-à-dire un prix tel que le surplus cumulé des deux périodes (1/2- $p_{1}$ en moyenne pour la première et $9 / 32$ pour la seconde) soit égal à son utilité de réserve (ici 0 ). On obtient alors un prix de première période égal à $\frac{25}{32}>2-\sqrt{2}$ et un profit total de $\frac{17}{32}>\sqrt{2}\left(\frac{7}{4}-\sqrt{2}\right)$.

Le consommateur paye donc en première période un prix élevé pour avoir le droit non seulement de consommer le bien à la première période mais également de bénéficier d’un tarif préférentiel à la seconde période. 
Les programmes de fidélisation sont donc une façon à la fois d'élargir les instruments tarifaires des entreprises et de discriminer entre les consommateurs dans des situations dynamiques où ils sont ex-ante identiques.

Mais ce type de programme n'est pertinent que si deux conditions sont réunies : la discrimination doit être possible et le monopole doit s'engager sur ses prix à l'avance.

\subsection{Engagement tarifaire}

Il est donc intéressant de pousser l'analyse plus en détail dans cette direction et de se concentrer sur les problèmes d'engagement temporel du monopole. Pour cela, il est nécessaire de modifier le cadre précédent en supposant que les consommateurs connaissent leurs préférences - définies par les dispositions à payer - au début de chaque période mais qu'elles peuvent évoluer d'une période à une autre. ${ }^{6}$ Comme auparavant, les programmes de fidélisation tentent d'inciter à une consommation précoce, mais il s'agit de discuter plus précisément de la capacité du monopole à mettre efficacement en place de tels programmes, en particulier sa capacité à s'engager sur les prix futurs.

Supposons, à titre illustratif, que les préférences des agents sont indépendantes d'une période à une autre ${ }^{7}$. Ainsi, au début de la première période, un consommateur connait sa disposition actuelle à payer pour le bien mais il ne sait rien de ses dispositions futures. Un premier système de fidélisation consiste à proposer en première période un prix unique puis en seconde période des prix différents aux clients de la première période et aux autres. Ainsi, à la première période, chaque consommateur compare son utilité s'il achète aujourd'hui, utilité que l'on peut décomposer entre son utilité immédiate et son utilité espérée à la deuxième période, calculée avec le prix proposé pour les anciens clients, avec son utilité s'il n'achète qu'à la deuxième période, c'est-à-dire son

${ }^{6}$ Dans le cadre précédent des biens d'expérience, il n'est pas possible de parler des problèmes de discrimination ex-ante car tous les consommateurs sont identiques. La discrimination étant au cœur des problèmes d'engagement, il est nécessaire de changer le cadre d'analyse et de supposer que les agents sont différents ex-ante et donc connaissent leurs préférences.

${ }^{7}$ Nous reprenons les idées modélisées par Caminal [2012]. 
utilité espérée à la deuxième période, calculée maintenant avec le prix proposé aux nouveaux clients.

Dans ce cadre, ce sont les consommateurs attribuant une valeur élevée au bien qui vont acheter aujourd'hui alors que les autres attendront la seconde période. Pour déterminer les prix choisis par le monopole, il est nécessaire de différencier les cas en fonction du pouvoir d'engagement de l'entreprise, c'est-à-dire de sa capacité à choisir en première période les prix de première et de deuxième période et à ne pas modifier son offre de deuxième période quand l'occasion se présente.

Supposons tout d'abord que le monopole n'ait aucun pouvoir d'engagement, ce qui revient à considérer le cas où les prix sont choisis successivement à chaque période. A la deuxième (et ici dernière) période, le monopole a tout intérêt à utiliser son pouvoir de marché et à proposer le prix statique de monopole à l'ensemble des consommateurs, anciens comme nouveaux. Mais ce sera également le cas à la première période, puisque tous les agents anticipent le comportement du monopole en deuxième période et donc acheter en avance ne donne aucun avantage en deuxième période. Ainsi, si une entreprise ne peut pas s'engager aujourd'hui sur les prix futurs, aucun programme de fidélisation (crédible) ne peut être mis en place.

Supposons au contraire que le monopole ait un pouvoir d'engagement partiel, c'est-à-dire puisse s'engager en première période sur les prix de deuxième période de ses anciens clients mais pas sur les prix de ses futurs clients. Puisque le monopole ne s'engage pas sur le prix de deuxième période des nouveaux clients, ce prix va être égal au prix statique de monopole pour maximiser le revenu de deuxième période sur ce segment de marché. Comment choisir les autres prix ? II s'agit, comme dans l'approche initiale de cette section, de choisir simultanément les deux prix pour attirer les consommateurs et générer du surplus. Pour cela, le monopole va proposer un prix faible pour la deuxième période - en fait un prix égal au coût marginal de production pour assurer une création de surplus maximale - en contrepartie d'un prix initial plus élevé que le coût mais inférieur au prix statique de monopole. Comme dans le cas d'un tarif binôme (avec un système mixte composé d'un abonnement et d'un paiement en fonction de la quantité consommée), le prix de seconde période est choisi pour créer du surplus et celui de première période pour collecter ce surplus. Notons que, par rapport au cas sans engagement, non seulement la firme est gagnante - elle aurait pu choisir les mêmes prix mais elle ne l'a pas fait - mais c'est également vrai pour les consommateurs et donc l'ensemble de la société. En effet, cette nouvelle situation conduit à des prix plus faibles, plus de 
consommation et donc plus de surplus créé, dont bénéficient ici à la fois le monopole et les consommateurs.

On peut également s'intéresser au cas d'engagement total, c'est-à-dire à une situation où le monopole choisit initialement trois prix, le prix de première période, le prix de deuxième période de ses anciens clients mais également le prix de deuxième période de ses futurs clients. Pour inciter les consommateurs, en situation d'incertitude sur leurs goûts futurs, à acheter dès maintenant, le monopole a intérêt à les menacer d'un prix très élevé en deuxième période. Pour ceci, le monopole veut annoncer aujourd'hui un prix futur bien supérieur non seulement au prix proposé aux anciens clients mais également supérieur au prix statique de monopole. Même si une telle politique est optimale pour le monopole, elle est difficile à mettre en place. En effet, une fois la deuxième période arrivée, la firme aura tout intérêt à choisir comme avant le prix statique de monopole et puisqu'aucun contrat formel n'aura été signé sur ce prix (il est en effet peu réaliste de supposer que la firme se serait engagée en première période sur les prix destinés à ses clients futurs), il y a peu de limite à la remise en cause du prix initialement annoncé.

\subsection{Coupons de réduction}

Une façon pour le monopole de résoudre son problème d'engagement consiste non pas à s'engager en première période sur des prix de deuxième période pour les futurs consommateurs - un tel engagement étant difficile à tenir - mais à proposer en première période des coupons de réduction pour les achats futurs. Ainsi, à la première période, le monopole va juste choisir le prix de première période et la réduction à laquelle ses clients auront droit par rapport à ses nouveaux clients en deuxième période. Cette réduction permet à la fois de garantir un avantage aux anciens clients et de lier les prix des anciens et des nouveaux clients en deuxième période. Ainsi, pour s'engager à choisir un prix très élevé pour les nouveaux clients en deuxième période, le monopole a intérêt à proposer des réductions très fortes pour ses anciens clients. De la sorte, pour gagner un peu d'argent sur ses anciens clients, le monopole devra proposer des prix très élevés sur les nouveaux clients. Notons que si les coupons de réduction permettent au monopole de résoudre ses problèmes d'engagement et donc d'augmenter ses profits, cela peut être au détriment des consommateurs par rapport au cas sans engagement car le prix des nouveaux clients va maintenant excéder le prix statique de monopole. 
Dans le cas où les valeurs attribuées par les consommateurs sont positivement corrélées entre les périodes, les mêmes mécanismes sont à l'œuvre avec d'un côté une volonté d'annoncer ex-ante des prix futurs différents pour les anciens et les nouveaux clients et de l'autre, une fois les premiers achats effectués, la tentation de baisser les prix de seconde période pour attirer le maximum de consommateurs et réaliser ainsi un profit additionnel. On reconnait ici le dilemme habituel d'un monopole dans le cas d'un bien durable (Coase [1972]) voulant s'engager à maintenir ses prix élevés pour inciter les agents attribuant une forte valeur au bien à consommer immédiatement mais qui, une fois ces achats effectués, ne peut s'empêcher de baisser les prix. Les consommateurs anticipant ce type de comportement vont donc attendre et s'abstenir de consommer immédiatement, ce qui conduit le monopole à proposer dès la première période des prix faibles pour tous (c'est la « conjecture de Coase » : voir Hart et Tirole [1988]). La différence est que dans le cas des biens durables, les consommateurs retardent ou non un achat unique alors que dans le cas des programmes de fidélité, le consommateur achète ou non en tenant compte des gains induits sur ses achats futurs. Dans ce cadre où les préférences temporelles sont fortement corrélées, les coupons de réduction permettent à nouveau de résoudre ce problème d'engagement. En choisissant un prix initial et en proposant un coupon de réduction assez élevé, la firme s'engage de façon crédible à conserver des prix élevés dans le futur pour les nouveaux clients, incitant de la sorte les clients ayant une valeur initiale élevée à consommer dès la première période.

Les programmes de fidélisation sont une façon d'inciter à la consommation dans un cadre où les consommateurs sont incertains sur leurs préférences futures pour le bien. Ils constituent un outil de discrimination entre consommateurs, et permettent à la firme d'extraire une plus grande partie du surplus créé par l'échange, mais sont également parfois susceptibles, en augmentant le nombre d'outils disponibles, d'augmenter l'efficacité de cet échange, c'est-à-dire la taille de ce surplus. Notons pour finir que les programmes de fidélisation les plus intéressants pour les entreprises nécessitent une forte capacité d'engagement. La mise en place d'un système de coupons de réduction est un bon moyen d'augmenter cette capacité.

\section{Fidélisation et concurrence}


Les pratiques de fidélisation, si elles sont parfois une façon d'inciter les consommateurs à consommer un bien inconnu d'eux, peuvent plus généralement se concevoir comme un instrument tarifaire dans la concurrence que plusieurs firmes se livrent sur un marché.

\subsection{Préférences et technologies}

Dans cet environnement concurrentiel, nous supposons que les consommateurs connaissent leurs goûts (présent et futur) pour le bien et les firmes utilisent les pratiques de fidélisation pour les attirer lors de leur premier achat ou bien éviter qu'ils ne décident de changer de fournisseur.

Dans un cadre dynamique, plusieurs raisons expliquent que les clients puissent être fidèles à leur fournisseur. Une première raison, particulièrement pertinente dans le cas des télécoms, est liée à la présence de switching costs ou coûts de changement (nouveau numéro, nouveau terminal, nouvelles habitudes). Dans cette configuration, les différentes entreprises présentes sur un même marché proposent des biens identiques mais il est coûteux de changer de fournisseur. Une seconde raison pouvant expliquer la fidélité des consommateurs est la présence de produits différenciés sur un même marché. Si les consommateurs ont des préférences intrinsèques pour un produit par rapport à un autre, il sera difficile pour les entreprises concurrentes de conquérir ces clients.

La présence de coûts de changement comme celle de biens différenciés justifient la mise en place de politiques tarifaires spécifiques, avec en particulier des prix en fonction des comportements antérieurs. Mais la perspective de prix différenciés ex-post va également influencer les comportements des consommateurs ex-ante et donc la concurrence que se livrent les différentes entreprises sur le marché. Nous verrons que les prédictions économiques dépendent largement du cadre considéré, à savoir le choix entre un modèle à coûts de changement et un modèle à produits différenciés.

\subsection{Concurrence et coûts de changement : le modèle pay-to-switch}

Une première façon d'analyser la concurrence entre entreprises consiste à s'intéresser à une situation où deux entreprises ( $\mathrm{A}$ et $\mathrm{B}$ ) proposent des biens en tous points identiques à chaque période. Les consommateurs attribuent tous la même valeur aux biens proposés mais, à la fin de la première période, ils doivent payer un coût s'ils décident de changer de fournisseur à la seconde 
période. Dans cette approche développée par Chen [1997], ce coût représente à la fois le temps passé en formalités, en comparaison des différentes offres mais également éventuellement la difficulté à renoncer aux anciennes habitudes. Pour des consommateurs timorés, ce coût sera élevé. II sera faible pour d'autres, curieux de nouveauté.

Pour bien comprendre ce type de concurrence, il est utile de commencer par réfléchir au comportement des entreprises lors de la deuxième (et ici dernière) période, c'est-à-dire une fois que les consommateurs auront pris leurs habitudes avec un des fournisseurs ${ }^{8}$. A ce stade, il y a en fait deux segments de marché, en fonction des décisions d'achat lors de la première période : le marché des anciens clients de A et celui des anciens clients de B. L'entreprise A (par exemple) va donc proposer des prix différents à ses anciens clients, qui doivent supporter un coût pour aller chez le concurrent, et aux clients de son concurrent, pour lesquels le changement de fournisseur est coûteux et qu'il est nécessaire de "subventionner ". L'entreprise A va proposer un prix élevé à ses clients, en prenant le risque d'en perdre quelques-uns (ceux dont les coûts de changement sont les plus faibles), de façon à préserver sa marge sur ces clients. Le prix proposé aux clients de la firme B va au contraire être faible pour attirer ces consommateurs, ou au moins ceux dont le coût de changement est faible. Ainsi, les prix d'équilibre vont provoquer de la mobilité d'une période à l'autre et les consommateurs " loyaux » feront face à des prix plus élevés que ceux proposés aux nouveaux consommateurs.

Si les consommateurs captifs permettent aux firmes de capitaliser en seconde période (le temps des "moissons"), les entreprises vont être prêtes à proposer des prix attractifs en première période (le temps des "semailles "). En effet, si tout client acquis en première période rapporte un bénéfice moyen $b$ en seconde, les entreprises seront disposées à diminuer leur marge d'autant en première période, et donc, si le bien a un coût marginal $c$, à proposer un prix $p=c-b$. Autrement dit, la concurrence entre firmes en première période conduit à un prix tel que, suite à une variation de part de marché, les gains et les pertes s'équilibrent entre les périodes. Et les prix facturés aux consommateurs - les loyaux comme les nouveaux - seront croissants dans le temps.

Comment comparer cette situation avec le cas sans discrimination, c'est-à-dire avec une situation où les mêmes conditions sont proposées aux anciens et aux nouveaux clients ? On peut montrer que les entreprises préféreraient que la discrimination soit interdite. En effet, en l'absence de

\footnotetext{
${ }^{8}$ On suppose que le marché est totalement couvert, c'est-à-dire que tout le monde achète à chaque période.
} 
discrimination, la concurrence serait moins intense en seconde période, les entreprises ayant moins d'instruments à leur disposition, et le prix serait plus élevé que les deux prix proposés dans le cas avec discrimination. L'analyse de la première période conduit à la même conclusion car, en l'absence de possibilité de moissonner en deuxième période, la demande est moins réactive aux variations de prix et la concurrence est plus faible. La discrimination est donc défavorable aux entreprises. Dans ce cadre où les baisses de prix ne génèrent pas de demande supplémentaire, la discrimination est également néfaste socialement car elle engendre des changements inefficaces (puisque les biens sont tous identiques) et donc des coûts pour l'ensemble de la société.

Ainsi, lorsque les entreprises proposent des produits identiques à des consommateurs captifs, les consommateurs loyaux sont très défavorisés par rapport aux nouveaux consommateurs, les entreprises adoptant des stratégies pay-to-switch

En réalité, il est clair que les consommateurs ne sont pas tous captifs de la même façon. En effet, si certains sont effectivement rétifs au changement (d'habitude, de comportement), d'autres sont prompts à changer de fournisseurs en cas de nouvelle opportunité. Biglaiser et al. [2012] s'intéressent à cette situation et montrent que, paradoxalement, la présence de consommateurs à faibles coûts de changements rend la concurrence plus faible sur le segment des consommateurs à forts coûts de changements. En effet, puisqu'il n'est pas possible de différencier les consommateurs en fonction de leurs coûts de changement propres, une offre avantageuse visant à attirer les consommateurs les moins mobiles va également attirer les consommateurs les plus mobiles. Mais de tels consommateurs sont susceptibles de repartir chez un concurrent en cas d'offre attractive à la période suivante, réduisant de ce fait leur valeur réelle. Cela conduit les firmes à proposer des offres moins agressives aux clients de leurs concurrents. Ainsi l'existence d'hétérogénéité dans les coûts de changement conduit à renforcer le rôle de ces coûts dans la fidélisation des clients.

\subsection{Concurrence et biens différenciés : le modèle pay-to-stay}

Au-delà des coûts de changement, la présence de produits différenciés sur un même marché peut donner des avantages à une entreprise sur une autre pour conquérir ou conserver une partie de sa clientèle. D'une façon générale, l'acte d'achat d'un consommateur révèle en partie ses goûts, ou au moins une préférence relative pour les biens produits par l'entreprise choisie. En l'absence de concurrence, l'entreprise choisirait des prix plus élevés pour les clients qui ont montré un intérêt 
pour ses produits car ces consommateurs ont probablement une élasticité de la demande plus faible. Mais en situation de concurrence, si un consommateur révèle sa préférence pour une marque en particulier, il incite les concurrents de cette marque à cibler ce client avec des offres plus avantageuses que ce qu'ils offrent en général. Ce comportement dit de "poaching » - ou braconnage - consistant à faire des offres très agressives aux clients de ses concurrents a deux conséquences. Tout d'abord, et d'une façon directe, un client engagé auprès d'un premier fournisseur peut bénéficier d'offres plus intéressantes qu'un nouveau client. Ensuite, une entreprise peut vouloir se protéger de la concurrence en proposant des programmes de fidélité.

L'analyse des programmes de fidélisation permet d'étudier le processus concurrentiel lorsque les entreprises utilisent I'historique des consommateurs ${ }^{9}$. On s'intéresse ici à une situation de marché avec deux entreprises ( $A$ et $B$ ) proposant des biens différenciés, et des consommateurs ayant une préférence stable dans le temps ${ }^{10}$ soit pour une entreprise, soit pour une autre. Il y a deux périodes d'échange et les entreprises peuvent librement utiliser les informations sur les choix passés des consommateurs.

Dans une situation symétrique (en termes de coût de production et de préférence des consommateurs), les deux entreprises vont proposer le même prix en première période et les consommateurs vont initialement choisir d'acheter à l'entreprise qu'ils préfèrent. C'est à la deuxième période que les entreprises vont proposer des offres ciblées d'un côté aux anciens clients et de l'autre aux nouveaux, c'est-à-dire aux consommateurs qui ont choisi leur concurrent en première période. En effet, l'entreprise $A$ sait que les consommateurs ayant acheté à $B$ en première période ont une préférence pour cette dernière firme donc elle leur propose une réduction de prix (un discount). L'entreprise B va faire de même avec les anciens clients de l'entreprise A, si bien que l'entreprise A, dans ses offres à ses propres clients, va arbitrer entre perdre des clients et augmenter ses marges sur les clients restants. Au final, l'entreprise A conquiert certains clients de B - ceux qui avaient une préférence peu marquée pour les produits de B - mais perd certains de ses

${ }^{9}$ Voir Fudenberg et Tirole [2000].

${ }^{10}$ Voir Chen et Percy [2010] lorsque les préférences peuvent changer d'une période à l'autre. 
propres clients - ceux ayant une préférence peu marquée pour les produits de $A$. Comme dans l'approche se focalisant sur les coûts de changement, à la seconde période les anciens consommateurs font face à des prix plus élevés chez leur fournisseur historique que chez son concurrent. Mais cette inertie est maintenant la conséquence d'une vraie préférence des consommateurs pour une des deux firmes. En première période, la concurrence va être assez faible entre les entreprises, plus faible qu'en l'absence de braconnage. En effet, comme les consommateurs savent qu'en choisissant une des deux entreprises en première période, ils recevront en seconde période une offre très intéressante de la part de l'autre, ils sont moins exigeants sur les offres de première période. En conséquence, les prix de première période seront plus élevés qu'en l'absence de braconnage.

On peut à nouveau analyser l'impact de la discrimination tarifaire de seconde période basée sur le comportement de première période. On a vu que le braconnage conduit à une concurrence plus vive en seconde période, et donc à des prix plus faibles qu'en l'absence de discrimination, mais également à une concurrence plus faible en première période, avec un prix plus élevé que sans discrimination. Plus précisément, le profit des entreprises diminue par rapport au cas sans discrimination et les consommateurs y gagnent (pour ceux qui changent de fournisseurs) ou sont indifférents. Au total, dans une situation où le marché est totalement couvert aux deux périodes, interdire la discrimination basée sur le comportement augmente le surplus agrégé en évitant les changements de fournisseurs inefficaces. Notons que la discrimination tarifaire est néfaste pour les entreprises car elle augmente les possibilités de concurrence entre les deux entreprises.

\subsection{Récompense ou punition des anciens consommateurs ?}

Dans un univers concurrentiel, l'attitude d'une entreprise vis-à-vis de ses consommateurs passés peut fortement varier. Comme on l'a vu, les modèles avec coûts de changement prévoient des prix croissants dans le temps pour les consommateurs loyaux alors que les modèles de biens différenciés prévoient une baisse de prix pour les consommateurs lors de leur deuxième période d'achat. Ainsi, deux types de stratégies existent : celle visant à récompenser la fidélité et l'autre visant principalement à attirer les nouveaux consommateurs. Cette coexistence peut même être observée à l'intérieur d'un même marché. C'est ainsi que le développeur d'antivirus McAfee a 
proposé en 2010 un rabais pour les nouveaux consommateurs alors qu'un de ses concurrents, Bitdefender, adoptait la stratégie inverse en récompensant les consommateurs fidèles.

Pour comprendre la coexistence de ces deux stratégies, il est intéressant d'étendre l'analyse à plus de 2 périodes en supposant que les consommateurs sont toujours présents deux périodes sur le marché mais que les entreprises le sont pour un nombre bien plus élevé de périodes ${ }^{11}$.

II y a donc maintenant trois types de consommateurs sur le marché : ceux qui n'achètent qu'à l'entreprise A, ceux qui n'achètent qu'à l'entreprise B, et les versatiles - ou surfers- qui achètent à l'entreprise proposant le prix le plus bas. Notons que dans ces trois cas, les consommateurs n'achètent que si le prix est inférieur à la valeur du bien pour eux, valeur que l'on suppose connue et uniforme dans la population. A chaque période, les entreprises font face à deux groupes de consommateurs. D'un côté, le groupe qui a acheté leur produit à la période précédente et de l'autre, le groupe qui ne l'a pas acheté, ce groupe étant composé à la fois d'anciens acheteurs du produit concurrent et de nouveaux consommateurs.

Dans ce cadre, on peut montrer que les deux entreprises vont adopter des stratégies inversées. Plus précisément, une entreprise, en proposant à des consommateurs qu'elle ne connait pas un prix égal à la valeur (identique pour tous) du bien pour ces consommateurs, est capable de sélectionner parmi les nouveaux consommateurs uniquement ceux qui lui sont toujours loyaux. Elle peut de cette façon les identifier et ainsi extraire également le maximum de leur surplus à la période suivante. En proposant un prix plus bas pour les nouveaux consommateurs, l'autre entreprise attire non seulement les consommateurs appréciant son produit mais également les consommateurs privilégiant l'entreprise la moins chère. A la seconde période, cette firme ne va pas pouvoir proposer le prix le plus élevé à ce groupe car l'autre firme va tenter de récupérer une partie de ces consommateurs opportunistes. On aura donc deux types de stratégie possibles. L'entreprise ayant identifié ses anciens clients comme des clients loyaux va leur proposer des prix élevés mais proposer des prix bas pour les autres clients. Au contraire, l'entreprise n'ayant pas initialement identifié ses clients loyaux propose des prix plus faibles à ses anciens clients (en grande partie des surfers) qu'aux nouveaux clients. Et les deux entreprises vont alterner entre ces deux stratégies, en choisissant toujours des stratégies inversées.

\footnotetext{
${ }^{11}$ Voir le travail de Caillaud et De Nijs [2011].
} 
Notons que si une entreprise propose un prix élevé aux nouveaux clients, un client loyal à cette entreprise pourrait décider de ne pas consommer en première période pour bénéficier d'un prix avantageux en deuxième période. L'entreprise va donc être contrainte de baisser un peu son prix et donc de laisser une partie du surplus de l'échange à ses consommateurs loyaux - mais les stratégies décrites ci-dessus restent pertinentes pour peu que les consommateurs escomptent suffisamment le futur.

\section{Applications}

L'objectif de cette section est de prolonger les analyses précédentes par des problématiques plus appliquées et plus directement liées aux débats actuels. Nous discuterons les enjeux liés aux contrats de long terme mais également le rôle des programmes de fidélité comme outil de coordination des firmes ainsi que l'aspect pro ou anti-concurrentiel des offres de rétention.

\subsection{Contrats de long terme}

Il s'agit ici d'analyser l'impact des contrats de long terme sur la concurrence. Les contrats de long terme donnent la possibilité aux acheteurs et aux vendeurs de s'engager à l'avance sur les quantités et les prix liés aux échanges futurs. II s'agit donc bien d'une forme de fidélisation par l'intermédiaire d'un engagement réciproque.

Les contrats de long terme - et leur caractère pro ou anti-concurrentiel - font l'objet d'un vaste débat dans la littérature économique. Une première approche - développée par les économistes de Chicago comme Bork [1978] ou Posner [1976] - affirme que les contrats de long terme ne sont pas des entraves à la concurrence mais introduisent dans la relation d'échange des clauses contraignantes utiles pour augmenter l'efficacité des relations économiques. En effet, en présence d'incertitude, l'opportunisme des agents rend plus difficile les investissements des différentes parties concernées par une transaction. En garantissant une durée suffisante à la relation économique (par contrat ou en allant jusqu'à l'intégration verticale quand il s'agit de clients industriels), les incitations à investir dans des actifs matériels ou humains relativement spécifiques augmentent, ce qui accroît le surplus social associé à la relation économique. Le contrat de long 
terme, en pérennisant une relation commerciale, pousse les parties à investir en actifs spécifiques de façon efficiente, donc à accroître la valeur du surplus total qu'elles se partagent.

Mais pour tirer des conclusions positives en termes de bien-être collectif des intuitions précédentes, il faut supposer que toutes les parties concernées par l'accord sont susceptibles de négocier. Lorsque ce n'est pas le cas, et si cet accord a un effet sur des agents qui ne sont pas parties prenantes à l'accord, les contrats de long terme peuvent être socialement néfastes, notamment par leurs effets anticoncurrentiels. En particulier, un contrat de long terme entre une firme et un consommateur permet à ces deux parties, en cas d'arrivée potentielle d'une firme plus efficace sur le marché, de capturer une partie des gains d'efficacité liés à l'arrivée de ce nouvel entrant. Ainsi le contrat de long terme peut être vu comme un instrument d'extraction de rente, non pas au détriment des consommateurs, mais au détriment des entrants potentiels et au bénéfice de la «coalition» formée de l'entreprise initialement en place et des consommateurs.

Illustrons ce point en analysant une situation où une entreprise $(A)$ en place fait face à l'entrée possible d'une entreprise concurrente $(B)^{12}$. Les biens produits par les deux entreprises sont évalués de la même façon par le consommateur représentatif mais, à l'étape initiale, alors que le coût de $A\left(c_{A}\right)$ est connu, celui de $B\left(c_{B}\right)$ n'est pas encore certain, la firme entrante pouvant être plus ou moins efficace que la firme en place. La concurrence se déroule en deux temps : en première période, l'entrant observe son coût et décide ou non de rentrer; en deuxième période, il y a production et vente. En l'absence de contrat de long terme, le consommateur attendrait la réalisation du coût de $B$ et, les deux entreprises se livrant une concurrence en prix, obtiendrait le bien à un prix égal au coût de la firme la moins efficace, $c^{\prime}$ est-à-dire $c_{A}$ si la firme $B$ est plus efficace et $C_{B}$ dans le cas inverse. Supposons maintenant que la firme $A$ ait la possibilité de signer un contrat avec le consommateur avant que le coût de B soit connu. Ce contrat va à la fois spécifier un prix d'achat et une pénalité à payer par le consommateur en cas de rupture du contrat. Cette pénalité va en réalité jouer le rôle d'un coût d'entrée pour la firme $B$ qui, si elle veut attirer le consommateur, devra lui verser une compensation au moins égale à la pénalité. Si on comprend bien l'intérêt pour A de proposer ce contrat, il est moins intuitif de voir l'intérêt pour le consommateur. En réalité, ce contrat permet d'améliorer son pouvoir de négociation vis-à-vis de la

\footnotetext{
${ }^{12}$ Voir l'article de Aghion et Bolton [1987].
} 
firme B lorsque celle-ci est la plus efficace car dans ce cas elle est contrainte de proposer un prix très faible.

\section{Contrat de long terme et barrière à l'entrée : Illustration numérique}

Pour illustrer plus précisément le rôle des contrats de long terme, supposons que le consommateur représentatif attribue une valeur de 1 au bien, que le coût marginal de $A, c_{A}$, est égal à $1 / 2$ alors que $c_{B}$ est une variable aléatoire suivant une loi uniforme entre 0 et 1 .

En l'absence de contrat de long terme, le prix obtenu par le consommateur dépend de la décision d'entrée de la firme $B$. Si $c_{B}<1 / 2$, la firme $B$ entre sur le marché et le consommateur paye un prix de $1 / 2$ alors que si $c_{B}>1 / 2$, seule la firme $A$ est présente et choisit un prix de 1 pour extraire l'ensemble du surplus du consommateur. Dans cette configuration, la firme $A$ obtient un profit espéré de $1 / 4$, le consommateur un surplus espéré de $1 / 4$ alors que la firme B obtient un profit espéré de $1 / 8$.

Supposons maintenant que la firme A propose un contrat de long terme avec un prix $3 / 4$ et une pénalité de $1 / 2$ en cas de rupture. Dans ce cas, l'entreprise $B$ devra proposer un prix très faible $(1 / 4)$ pour que les consommateurs, malgré la pénalité, décident de changer de fournisseur. Elle ne rentrera donc sur le marché que si son coût est très faible, inférieur à $1 / 4$. Avec ce contrat, la firme ayant proposé le contrat de long terme va obtenir un profit espéré de $5 / 16$, la firme $B$ obtenant $1 / 32$ alors que le consommateur obtient exactement le même surplus espéré qu'en l'absence de contrat. Comme on le voit, le contrat de long terme permet d'augmenter le profit espéré de la firme $A$ mais induit une perte de surplus social car la firme qui produit n'est pas toujours la plus efficace.

Ce contrat de long terme permet donc à l'entreprise A de limiter l'impact de la concurrence car seules les entreprises beaucoup plus efficaces qu'elle pourront entrer. Quant aux consommateurs, ils ne perdent rien car cette pénalité leur permet d'obtenir un prix beaucoup plus faible lorsque $B$ entre effectivement sur le marché. Notons que le résultat dépend fortement de l'hypothèse que la firme B n'est pas présente à la période initiale et donc ne peut pas proposer un contrat concurrençant celui proposé par la firme A (par exemple une offre de récompense si le consommateur ne signe pas de contrat ex-ante). 
L'idée qu'une entreprise en place, bien souvent un monopole historique, peut utiliser des contrats de long terme pour bloquer l'entrée a été généralisée à d'autre cadres, en particulier au cas où la présence d'économies d'échelle donne un avantage important aux entreprises possédant une large base installée ${ }^{13}$. Mais dans cette approche traditionnelle des contrats de long terme, seule une des entreprises peut proposer de tels contrats et donc bénéficie d'un véritable avantage stratégique. Dans des marchés plus matures, les contrats de long terme peuvent être proposés par l'ensemble des firmes. L'article de Fubenberg et Tirole [2000] - déjà évoqué plus haut- envisage cette possibilité en supposant que les entreprises peuvent proposer, en plus des contrats de court terme, des contrats valables pour les deux périodes.

On suppose ici que deux entreprises (A et B) sont en concurrence pour des consommateurs différenciés par leurs préférences intrinsèques plus ou moins marquées pour $A$ et $B$. Notons que ces contrats de long terme permettant de soustraire leurs clients à la concurrence, les entreprises pourraient être tentées de ne proposer que des contrats de ce type. Mais en faisant cela, elles dissuaderaient certains clients potentiels. II est finalement préférable, pour attirer certains types de clients, de leur laisser la liberté de changer de fournisseur en deuxième période et donc proposer également des contrats de court terme.

Si les entreprises A et B proposent des contrats de court terme et des contrats de long terme, l'ensemble des consommateurs - différenciés initialement par leurs préférences plus ou moins fortes pour l'entreprise A et B - va être divisé en quatre groupes. Ceux ayant une préférence forte pour A vont choisir le contrat de long terme proposé par A, ceux ayant une préférence plus faible pour $A$ mais préférant $A$ à $B$ vont choisir le contrat de court terme proposé par $A$, ceux préférant faiblement B à A vont choisir le contrat de court terme proposé par B et ceux ayant une préférence marquée pour B choisiront le contrat de long terme proposé par B.

Au début de la deuxième période, chaque entreprise ne s'intéresse plus qu'aux groupes intermédiaires, composés des clients surfers, les deux groupes extrêmes étant de facto sortis du marché, et comme dans le cas sans contrat long terme, des offres différenciées sont proposées à ces deux groupes. La présence initiale de contrat de long terme va néanmoins engendrer quelques changements. Tout d'abord, l'offre faite par une firme à ses clients de première période sera plus agressive. En effet, comme ces clients sont les plus réceptifs aux offres de son concurrent, et qu'ils

\footnotetext{
${ }^{13}$ Voir Rasmusen, Ramseyer et Wiley [1991] et Segal et Whinston [2000].
} 
ne représentent qu'une partie de sa clientèle, il est optimal de leur proposer des prix plus faibles. Ensuite, comme la perspective d'une concurrence forte en deuxième période peut dissuader les consommateurs de choisir initialement des contrats de long terme, les entreprises vont être contraintes de proposer des prix bas également pour ces types de contrats. Par conséquent, les profits des entreprises vont être plus bas que si les contrats de long terme n'étaient pas autorisés. Notons également qu'une partie des consommateurs va changer de fournisseur d'une période à une autre mais que ces changements - inefficaces car certains consommateurs ne choisissent pas l'entreprise dont ils sont les plus proches - seront plus rares.

Au final, la présence de contrats de long terme, en créant une forme implicite de différenciation entre les clients loyaux et les clients versatiles, augmente le nombre d'outils de concurrence, l’intensité de celle-ci et conduit à un bien-être plus élevé.

\subsection{Les programmes de fidélisation comme outil de collusion}

Une des questions liées aux programmes de fidélisation touche à la possibilité d'utiliser de tels programmes pour organiser une entente entre les entreprises d'un secteur donné. Cette entente peut être explicite mais nous ne considérons ici que le cas d'une entente implicite. Autrement dit, il s'agit de voir si certaines formes de fidélisation ne permettent pas aux firmes, dans un cadre pourtant concurrentiel, d'obtenir les mêmes niveaux de profit que dans un cadre monopolistique. Ackermann [2010] souligne par exemple le rôle potentiellement collusif des rabais proposés exante pour des achats futurs. Plus précisément, concentrons-nous sur une situation de concurrence entre deux firmes identiques, $A$ et $B$, faisant face de façon répétée à des consommateurs tous identiques. A la première période, chaque firme propose un prix ainsi qu'un rabais à valoir en seconde période pour ses consommateurs de première période. A la seconde période, chaque firme choisit son prix et les consommateurs, en fonction de ces prix et des rabais éventuels, font leur choix de produits. Notons que si un consommateur n'ayant pas acheté à la firme A (ou B) en première période ne peut prétendre l'avoir fait, l'inverse n'est pas vrai si bien qu'un consommateur peut toujours se faire passer pour un nouveau consommateur, quel que soit son historique réel de consommation.

Supposons que le marché est partagé au début de la deuxième période, la moitié des consommateurs ayant initialement choisi l'entreprise A et l'autre moitié ayant préféré l'entreprise 
B. Chaque firme, qui ne peut proposer qu'un seul prix à l'ensemble des consommateurs quelles que soient leurs consommations passées, a alors le choix entre deux stratégies : soit proposer un prix élevé pour exploiter ses clients passés, soit proposer un prix bas pour attirer en plus des consommateurs ayant choisi l'autre firme à la première période. Si les rabais accordés initialement sont suffisamment élevés, chaque firme va se concentrer sur ses clients passés. Autrement dit, en deuxième période, chaque firme va proposer un prix élevé, en réalité égal au prix de monopole auquel on ajoute le rabais accordé à la première période. Puisque la concurrence est inexistante en première période, les firmes peuvent également se coordonner sur des prix élevés en première période. Ainsi, en proposant conjointement des discounts en première période, chaque entreprise rend le coût de la concurrence très élevé et dissuade l'autre entreprise d'attirer ses clients. Les rabais, s'ils sont conséquents, permettent aux entreprises de se partager les profits de monopole, et donc le surplus de la relation d'échange, au détriment des consommateurs.

II faut tout de même tempérer l'ampleur des distorsions potentielles créées par ces politiques de fidélisation. Tout d'abord avec plus de deux firmes, les gains de la collusion sont plus faibles - car le profit de monopole doit être partagé entre plus de firmes - ce qui complexifie les stratégies de coordination. Ensuite, dans un cadre réellement dynamique où de nouveaux consommateurs apparaissent à chaque période, les firmes vont se faire concurrence pour attirer ces nouveaux consommateurs ce qui dissuade de proposer des discounts importants et de ce fait limite les possibilités de coordination entre entreprises ${ }^{14}$. Ainsi dans des marchés très changeants, et avec beaucoup d'entreprises en concurrence, le rôle anti-concurrentiel des rabais accordés aux anciens clients est à nuancer.

\subsection{Les offres de rétention}

La concurrence croissante dans les services et le développement de biens incluant des systèmes d'abonnement ont modifié les pratiques concurrentielles de nombreuses entreprises. Ainsi, non seulement les firmes font des efforts pour démarcher les clients ayant souscrit des offres chez leurs concurrents mais elles mettent également en place des politiques spécifiques visant à conserver leurs propres clients. Par exemple, dans les services bancaires, internet ou téléphoniques, il est

\footnotetext{
${ }^{14}$ Sur ces deux points, voir Caminal et Claici [2007].
} 
courant qu'un individu annonçant sa volonté de changer de fournisseur se voie proposer une offre spécifique, c'est-à-dire un rabais de façon à diminuer l'attractivité de la firme concurrente. Cette pratique s'est généralisée au point que certaines entreprises possèdent même des services spécialement consacrés à la fidélisation des consommateurs. S'agissant de retenir les candidats au départ, on parlera d'offre de rétention.

Ces comportements, désignés dans la littérature anglo-saxonne sous le terme de save activity ou encore de retention offers, font l'objet de débats académiques mais aussi entre les acteurs des différents secteurs concernés, y compris leur autorités régulatrices. Sous ces débats se trouvent la question de l'intérêt pour une firme entrant sur un marché de proposer une offre concurrentielle à une partie de la population si le fournisseur traditionnel a la possibilité à la fois de prendre connaissance de l'existence de cette offre mais également de formuler une contre-offre. Comme nous allons le voir, le caractère pro ou anti-concurrentiel de ces activités de rétention va fondamentalement dépendre des caractéristiques des consommateurs, et plus particulièrement du type d'hétérogénéité existant entre ces consommateurs.

Nous supposons d'abord que les consommateurs sont principalement différenciés par leurs coûts de changement. Ensuite nous considérons la possibilité pour les firmes d'offrir non seulement un prix à leurs anciens clients et un prix aux clients de ses concurrents mais également un prix de rétention à ses clients ayant reçu une offre concurrente. Enfin, il est intéressant de suivre Gnutzmann [2012] en supposant que le coût de changement de fournisseur est scindé en deux parties, une première payée pour aller effectivement chercher une nouvelle offre et une deuxième partie uniquement payée si le changement de fournisseur est effectif.

L'existence d'offres de rétention modifie la concurrence à la première comme à la seconde période. A la seconde période, la concurrence est accrue. En effet, puisque seuls les consommateurs ayant des coûts de changement faibles vont solliciter des offres concurrentes, la population concernée est assez homogène ce qui conduit à une concurrence plus vive et donc à des offres de rétention très concurrentielles. La présence de ces offres avantageuses conduit également à baisser les prix proposés aux consommateurs loyaux pour les dissuader de rechercher des offres concurrentes (et donc éventuellement de bénéficier d'offres de rétention très attractives). Enfin, la difficulté de conquérir des parts de marché en deuxième période incite les entreprises à proposer des prix attractifs également en première période. 
Ainsi, dans un cadre où les consommateurs sont différenciés par leurs coûts de changement et où une partie de ce coût est payée avant la finalisation du changement de prestataire, la présence des offres de rétention augmente la concurrence à la seconde période et en conséquence à la première. Cela conduit à des profits plus faibles mais également à des changements effectifs de fournisseurs moins importants, et donc à une amélioration nette du bien-être social.

Mais comme évoqué plus haut, ce raisonnement n'est valable que dans le cas où les coûts de changement sont la principale dimension d’hétérogénéité entre les consommateurs. Dans les autres cas, l'impact des offres de rétention est plus discutable et fait l'objet de débats animés. C'est ainsi que l'Ofcom, régulateur britannique des télécoms, a émis de fortes réserves sur la validité de telles offres et plus globalement sur l'ensemble des pratiques de save activity ${ }^{15}$. L'argument principal développé dans les différents rapports tient à l'aspect anti-concurrentiel des pratiques de garantie de prix les plus bas, les offres de rétention pouvant s'assimiler à ce type de pratiques tarifaires. Plus précisément, ces offres, en donnant plus de possibilités aux firmes dominantes de garder leurs clients, dissuadent les concurrents potentiels de proposer des offres aux clients de ces firmes. De plus, les firmes dominantes ont un avantage informationnel quant à la profitabilité des clients, et donc à l'opportunité de faire des offres de rétention. Ainsi, ces offres conduisent en réalité les entrants à se contenter au mieux des clients les moins profitables, ce qui diminue également leur incitation à participer au marché.

Notons que si les régulateurs voient dans les taux élevés de changements de fournisseurs (le taux de brassage, ou churn) un signe de vitalité concurrentielle et donc un élément positif, les modèles économiques sont beaucoup plus réservés. Cette différence de perception est due en grande partie à l'hypothèse d'homogénéité des produits qui fait de tout changement de fournisseurs une simple réallocation sans aucune création de valeur.

Comme on le voit, l'aspect pro et anti-concurrentiel des offres de rétention dépend de la situation réelle, et en particulier de la nature des différences entre consommateurs. Si les consommateurs ont la même structure de consommation (c'est-à-dire la même profitabilité pour les firmes) mais diffèrent par leur coût de mobilité, les offres de rétention ont tendance à augmenter la concurrence. Si au contraire les consommateurs sont principalement différenciés par leur structure

\footnotetext{
${ }^{15}$ Voir le rapport Ofcom [2010].
} 
de consommation, cela donne un avantage important à la firme dominante, rendant de ce fait les offres de rétention anti-concurrentielles.

\section{Conclusion}

Les pratiques de fidélisation peuvent prendre des formes très diverses - ticket de réduction pour les achats futurs, chèque cadeau en fonction des achats passés, incitation à la signature de contrats de long terme - et constituent un outil commercial très attractif à la disposition des entreprises. Les conséquences réelles de telles pratiques dépendent largement du contexte, c'est-à-dire principalement de la structure de marché, de son historique et des préférences des consommateurs.

Dans le cas d'un marché en monopole, les pratiques de fidélisation peuvent avoir deux objets. Tout d'abord, elles constituent une façon de discriminer entre consommateurs, de leur proposer des offres différentes en fonction de leurs caractéristiques. En s'engageant à proposer des prix futurs différenciés entre anciens et nouveaux consommateurs, une entreprise induit une consommation immédiate plus importante. Ensuite, ces pratiques sont une façon de limiter l'entrée de nouveaux concurrents en rendant plus complexe les changements de fournisseurs pour les consommateurs. Dans ce cas, elles peuvent s'assimiler à la mise en place de coûts de changement de fournisseurs. Depuis l'arrêt Hoffman-La Roche (1979), les offres de rabais ou de fidélité proposées par les entreprises en position dominante sont particulièrement contrôlées par les autorités de la concurrence. Par exemple, I'issue des cas Michelin I (1993), Michelin II (2001), ou encore Orange Caraïbes (2012), a largement été influencée par la position dominante des entreprises mises en cause $^{16}$. Autrement dit, les mêmes offres proposées par des entreprises non-dominantes $\mathrm{n}^{\prime}$ auraient probablement pas été invalidées ${ }^{17}$. Ainsi, les offres de fidélisation comme outil simple de discrimination des consommateurs constituent, au même titre que les tarifs dégressifs par

\footnotetext{
${ }^{16}$ On pourra consulter les avis de l'Autorité de la concurrence sur le site www.autoritedelaconcurrence.fr.

${ }^{17}$ Voir Bouckaert et al. [2008] sur les effets des restrictions à la tarification différenciée d'une firme dominante.
} 
exemple, une pratique acceptable, capable de créer du surplus dans les relations entre firmes et consommateurs, potentiellement au bénéfice des uns comme des autres.

Lorsque le marché est plus concurrentiel, les effets de la fidélisation sont assez différents. Bien plus qu'une méthode de discrimination en fonction des préférences des consommateurs, ces pratiques sont un outil supplémentaire au service de la concurrence. En effet, la mise en place de pratiques de fidélisation conduira les entreprises à proposer des prix différents à leurs clients passés et aux clients ayant choisi les autres firmes. Notons que ce dernier groupe ayant choisi une autre entreprise initialement - soit par hasard, soit par goût - peut se voir proposer des tarifs très attractifs pour changer de fournisseur. II n'est donc pas toujours intéressant pour un consommateur d'être un "ancien " car celui-ci a plus de chance d'être considéré comme captif, même si en compensation, il a sans doute obtenu un avantage initial. Au total, il semble que dans le cadre d'un marché concurrentiel, les offres de fidélisation et plus globalement la discrimination tarifaire en fonction de l'historique de consommation accentue la pression concurrentielle.

Pour conclure, et contrairement à l'idée commune, les pratiques de fidélisation - et plus globalement l'utilisation des historiques de consommation - sont plutôt bénéfiques aux consommateurs. Elles permettent d'élargir les possibilités d'échanges et, en accentuant l'intensité concurrentielle, conduisent les entreprises à rétrocéder une grande partie des gains de l'échange. II est cependant important de s'assurer que toutes les entreprises présentes sur le marché ont la capacité de proposer des offres similaires pour que cette tarification ne permette pas la mise en place (ou la préservation) d'une position dominante.

\section{REFERENCES}

P. Ackermann [2010] Loyalty Rewards and Monopoly Pricing, WP Universität Bern.

P. Aghion et P. Bolton [1987] : Contracts as Barriers to Entry, American Economic Review, Vol. 77, pp. 388-401.

L. Basso, M. Clements et T. Ross [2009] : Moral Hazard and Customer Loyalty Programs, American Economic Journal: Microeconomics, Vol. 1, pp. 101-123.

G. Biglaiser, J. Crémer et G. Dobos [2012] : The Value of Switching Costs, à paraître dans Journal of Economic Theory.

R. Bork [1978] : Antitrust Paradox. Basic Book, New York. 
J. Bouckaert, H. Degryse et T. Van Dijk [2008] : Price Discrimination Bans on Dominant Firms, CESIfo WP n²192.

B. Caillaud et R. de Nijs [2011] : Strategic Loyalty reward in Dynamic Price Discrimination', WP $\mathbf{n}^{\circ}$ 29, Paris School of Economics.

R. Caminal [2012] : The Design and Efficiency of Loyalty Rewards, Journal of Economics and Management Strategy, vol. 21, pp. 339-371.

R. Caminal et A. Claici [2007] : Are loyalty-rewarding pricing schemes anti-competitive?, International Journal of Industrial Organization, Vol. 25, pp. 657-674.

Y. Chen [1997] : Paying Customers to Switch, Journal of Economic and Management Strategy, Vol. 6, pp. 877-897.

Y. Chen et J. Percy [2010] : Dynamic Pricing: when to entice brand switching and when to reward consumer loyalty, The RAND Journal of Economics, Vol. 41, pp. 674-685.

R. Coase [1972] : Durable Goods Monopolists, Journal of Law and Economics, vol. 15, pp. 143-150.

J. Crémer [1984] : The Economics of Repeat Buying, The RAND Journal of Economics, Vol. 15, pp. 396-403.

D. Fudenberg et J. Tirole [2000] : Customer Poaching and Brand Switching, RAND Journal of Economics, Vol. 31, pp. 634-657.

H. Gnutzmann [2012] : Paying Consumers to Stay, WP European University Institute, Florence.

O. Hart et J. Tirole [1988] : Contract Renegotiation and Coasian Dynamics, Review of Economic Studies, Vol. 55, pp. 509-40.

W. Hartmann et B. Viard [2008] : Do Frequency Reward programs Create Switching Costs?, Quantitative Marketing and Economics, Vol. 6, pp. 109-137.

P. Klemperer [1995] : Competition when Consumers have Switching Costs: An Overview with Applications to Industrial Organization, Macroeconomics, and International Trade, Review of Economic Studies, Vol. 62, pp. 515-39.

M. Lederman [2007] : Do Enhancements to Loyalty Programs Affect Demand? The Impact of International Frequent Flyer Partnerships on Domestic Airline Demand, The RAND Journal of Economics, Vol. 38, pp. 11341158.

L. Meyer-Waarden [2012] : Management de la fidélisation, Vuibert Editeur.

Ofcom, 2010. Strategic review of consumer switching: A consultation on switching processes in the UK communications sector, Consultation Document. 
M. Park [2011] : The Economic Impact of Wireless Number Portability, The Journal of Industrial Economics, Vol. 59, pp. 714-745.

R. Posner [1976] : Antitrust Law. University of Chicago Press, Chicago.

E. Rasmusen, J. Ramseyer et J. Wiley [1991] : Naked Exclusion, American Economic Review, Vol. 81, pp. 1137-1145.

I. Segal et M. Whinston [2000] : Naked Exclusion: Comment, American Economic Review, Vol. 90, pp. 296-309.

B. Viard [2008] : Do Switching Costs make Markets more or Less Competitive: the Case of 800Number Portability, the RAND Journal of Economics, Vol. 38, pp. 146-163. 